\title{
Erratum to: Several Real Variables
}

\author{
Shmuel Kantorovitz
}

\section{S. Kantorovitz, Several Real Variables, Springer Undergraduate Mathematics Series, DOI 10.1007/978-3-319-27956-5}

This book was inadvertently published without updating the following corrections:

$$
\begin{aligned}
& \text { Abbreviations: } \\
& \text { p. = page } \\
& \text { l. = line } \\
& \mathrm{fb}=\text { from bottom } \\
& \rightarrow=\text { should be replaced by }
\end{aligned}
$$

\section{Corrections:}

p.6, 1. before 1.1.6: omit "The conceptual importance"

p.12, (v): omit "(Fig. 1.1)"

p.12, (vi): omit "(Fig. 1.2)"

p.12, 1.6 fb: omit "(Fig. 1.3)"

p.12, 1.5 fb: omit "(Fig. 1.4)"

p.73, 1.17: "(2.17) in Theorem 2.1.5” $\rightarrow$ (2.10)

p.75, 1.13 fb: omit "in Theorem 2.1.12"

\footnotetext{
The updated original online version for these chapters can be found at DOI 10.1007/978-3-319-27956-5_1

DOI 10.1007/978-3-319-27956-5_2

DOI 10.1007/978-3-319-27956-5_3

DOI 10.1007/978-3-319-27956-5_4

DOI 10.1007/978-3-319-27956-5
}

S. Kantorovitz $(\bowtie)$

Bar-Ilan University, Ramat Gan, Israel

e-mail: shmuel.kantorovitz@gmail.com 
p.106, line before 3.2.6: $(2,-1) \rightarrow(3,-2)$

p.134, $1.11 \mathrm{fb}: 1.3 .3 \rightarrow$ "Proposition in Sect. 1.3.7"

p.137, $1.8 \mathrm{fb}: 3.6 .1 \rightarrow 3.6 .2$

p.162, last line: "Arcoli-Arzela" $\rightarrow$ "Arzela-Ascoli"

p.169, $1.9 \mathrm{fb}:(4.7) \rightarrow(4.11)$

p.171, 1. 3-4: "Example 4.1.5" $\rightarrow$ "Theorem 4.1.6"

p.194, 1.8: "Example" $\rightarrow$ "Examples"

p.246, 1.1: " $d(x, v)$ " $\rightarrow$ “ $d(x, y)$ "

p.256, 1.14: omit "below"

p.266, 1.16: "|Tu\|" $\rightarrow$ " $\|T u\| "$

p.290, lines 8-12: omit entirely.

p.290, 1.7: add the following: "letting $\epsilon \rightarrow 0+$, we conclude that the value of the given integral is $1 / 2 \pi-2 / \pi^{3}$." 